\section{Bromfenac as a Second Line Therapy for the Management of Post-Operative Cystoid Macular Oedema}

\section{Aditi Gupta ${ }^{1 *}$, Osama Makhzoum ${ }^{2}$, Anna Jane Brown ${ }^{3}$ and Rosemary Robinson ${ }^{2}$}

${ }^{1}$ Department of Ophthalmology, Birmingham and Midland Eye Centre, West Midlands, United Kingdom

${ }^{2}$ Department of Ophthalmology, University Hospital Coventry \& Warwickshire NHS Trust, Clifford Bridge Road, Coventry, West Midlands, United Kingdom

${ }^{3}$ Clinical Evidence Based Information Service (CEBIS) Specialist, University Hospital Coventry and Warwickshire NHS Trust, Clifford Bridge Road, Coventry, West Midlands, United Kingdom

\begin{abstract}
Purpose: To report case series of successful medical management of six patients diagnosed with post-operative Cystoid Macular Oedema (CMO).

Methods: A retrospective observational case series, which included patients with post-operative $\mathrm{CMO}$ where response to ketorolac was either minimal or unsustained and bromfenac was used as second line therapy.

Results: Four of these cases failed to show any improvement with ketorolac eye drops but showed a significant response to bromfenac. In one case, the response to ketorolac was unsustained but after instating bromfenac, a sustained and complete resolution of $\mathrm{CMO}$ was noted. In the last case described, an incomplete response to ketorolac was seen after six weeks, but with bromfenac, the patient reported a rapid response.

Conclusion: Switching from ketorolac to bromfenac with a different mechanism of action can be effective in resolving persistent post-operative $\mathrm{CMO}$ and may prevent the need for invasive treatment.

Keywords: Bromfenac; Ketorolac; Macular oedema; Optical coherence tomography; Post-operative
\end{abstract}

*Corresponding author: Aditi Gupta, Department of Ophthalmology, Birmingham and Midland Eye Centre, West Midlands, B18 7QH, United Kingdom, Tel: +44 7878991936; E-mail: draditig@gmail.com

Citation: Gupta A, Makhzoum O, Brown AJ, Robinson R (2015) Bromfenac as a Second Line Therapy for the Management of Post-Operative Cystoid Macular Oedema. J Ophthalmic Clin Res 2: 009.

Received: January 26, 2015; Accepted: March 03, 2015; Published: March 17,2015

\section{Introduction}

With the advent of Optical Coherence Tomography (OCT), Cystoid Macular Oedema (CMO) following cataract surgery or any other ocular procedure is being diagnosed earlier compared to previous years. With advancing surgical technology and skill, post-operative expectations regarding visual function are becoming higher. Early recognition and management of CMO is critical in helping patients to maintain their visual function. Post-operative CMO can become a clinical challenge and patients require constant reassurance in anticipation that a further surgical intervention may be needed.

The etiology of $\mathrm{CMO}$ is known to depend on upregulation of inflammatory mediators in the aqueous and vitreous humor following surgical procedure. Topical Non-Steroidal Anti-Inflammatory Drugs (NSAIDs) are potent inhibitors of prostaglandins and in conjunction with topical steroids have been the mainstay for the treatment of post-operative CMO [1,2]. Other options such as periocular or intravitreal steroids are usually considered if there has been no improvement after six weeks of topical NSAIDs.

Available topical NSAIDs for the management of post-operative CMO include diclofenac, ketorolac, bromfenac and nepafenac.

In our practice, ketorolac $0.5 \%$ eye drops (Acular, Allergan Limited, UK) four times a day (QDS) is used as first-line therapy for the management of CMO based on evidence and cost-effectiveness ratio. We considered using bromfenac as second-line NSAID as it was the next cost-effective drug.

In this case-series, we report successful medical management of six cases of post-operative CMO, of which five cases were pseudophakic $\mathrm{CMO}$ and one case was CMO following trabeculectomy procedure. None of these patients had received prophylactic Non-Steroidal Anti-Inflammatory Drugs (NSAIDs) for prevention of acute CMO. OCT images of two patients have been presented.

\section{Cases}

Case 1: A 76-year-old male underwent trabeculectomy with mitomycin $\mathrm{C}$ in his right eye. One week later, his visual acuity dropped from LogMAR 0.0 to 0.5 and Spectral Domain (SD) OCT detected signs of CMO. Central Foveal Thickness (CFT) measured $312 \mu \mathrm{m}$. Intra-ocular pressures were recorded as $16 \mathrm{mmHg}$ and there were no signs of hypotony. He was already on topical steroids following trabeculectomy and it was decided to add ketorolac QDS to manage CMO. Four weeks later, the patient's visual acuity dropped further to 0.6 and SD-OCT showed further increase in CMO (CFT-409 $\mu \mathrm{m})$. Ketorolac was stopped and bromfenac $0.09 \%$ (Yellox, Bausch \& Lomb UK Limited) eye drops twice a day (BD) was substituted. Four weeks later, his vision improved to 0.0 with complete resolution of $\mathrm{CMO}$ (CFT-212 $\mu \mathrm{m})$. At six months follow up, there has been no recurrence of $\mathrm{CMO}$ and visual acuity continues to remain 0.0 .

Case 2: An 82-year-old male underwent routine cataract surgery and developed CMO eight weeks following cataract surgery. His visual acuity dropped from -0.1 to $0.5(\mathrm{CFT}-356 \mu \mathrm{m})$. The patient was started 
on ketorolac and topical steroids. Six weeks later, there was no change in CFT on OCT imaging. Ketorolac was stopped and bromfenac eye drops BD were commenced. Four weeks later, his visual acuity improved to $-0.1(\mathrm{CFT}-206 \mu \mathrm{m})$ with complete resolution of CMO. At four months follow up; the patient continues to maintain good vision with no recurrence of CMO (Figure 1).

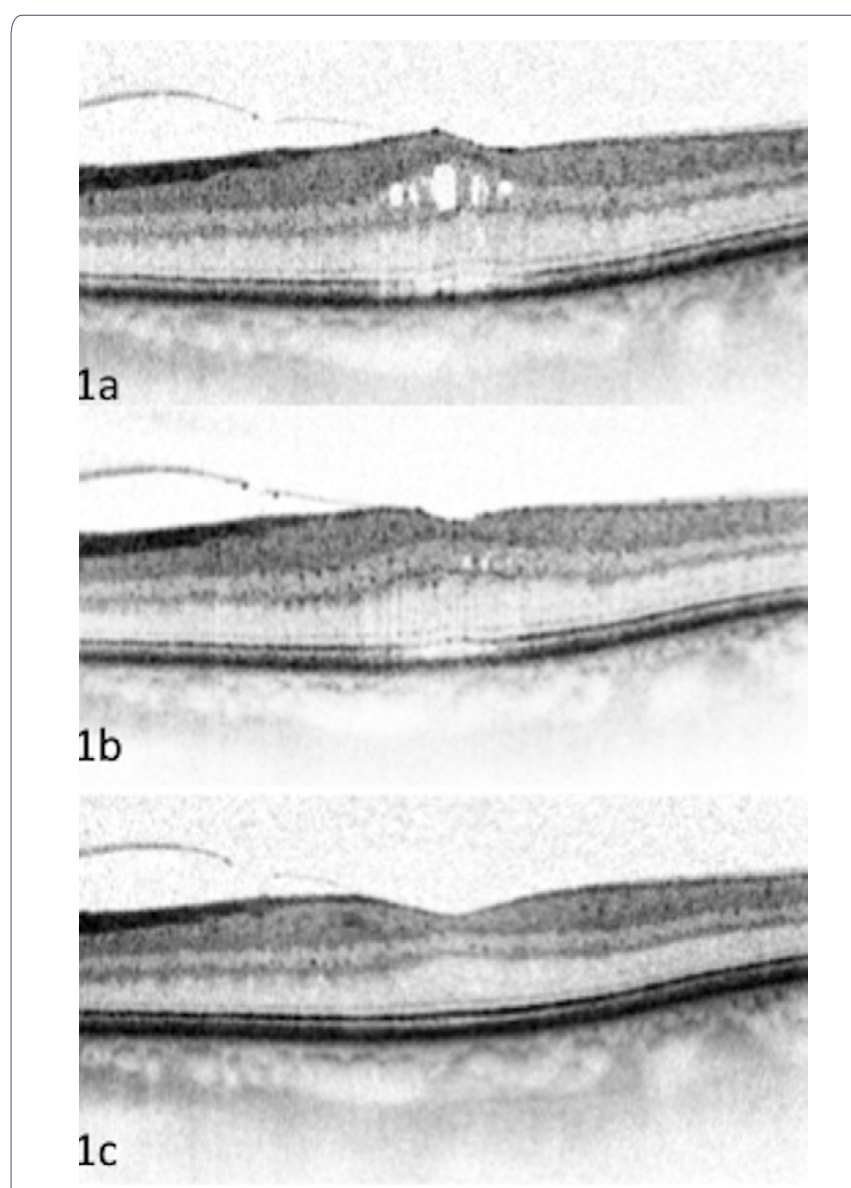

Figure 1: Post-operative CMO following routine cataract surgery.

a) $\mathrm{CMO}$ persisted following treatment with ketorolac. Bromfenac commenced (15 weeks post surgery).

b) 2 weeks following bromfenac treatment. OCT shows signs of resolving $\mathrm{CMO}$

c) 4 weeks later, complete resolution of $\mathrm{CMO}$ seen on bromfenac.

Case 3: A 70-year-old female with a history of Epi-Retinal Membrane (ERM) underwent routine phacoemulsification and developed post-operative CMO eight weeks later with a noticeable drop in visual acuity 1.0 (CFT-362 $\mu \mathrm{m})$. There was no response to combination of topical ketorolac and steroids at four weeks follow up. Bromfenac BD was substituted in place of ketorolac and four weeks later, her visual acuity improved to 0.3 (pre-op visual acuity was 0.4 ) and there was complete resolution of CMO seen on OCT (CFT-209 $\mu \mathrm{m})$.

Case 4: A 72-year-old female underwent right eye complicated phacoemulsification with anterior vitrectomy. She was known to have a mild ERM as in case 3 in the same eye. Pre-operatively, her visual acuity was 0.4 . Five weeks following complicated cataract surgery, she presented with a drop in visual acuity to 1.0 and OCT showed signs of CMO (CFT-334 $\mu \mathrm{m})$. She was started on ketorolac and topical steroids. Four weeks later, visual acuity improved to 0.4 and OCT showed decrease in CFT. 13 weeks following surgery, her best-corrected visual acuity was recorded as 0.5 and OCT continued to show signs of CMO. Ketorolac was then switched to bromfenac drops $\mathrm{BD}$ and four weeks later, her visual acuity improved to 0.2 with signs of nearly resolved CMO on OCT (CFT-201 $\mu \mathrm{m})$. At four months follow up; her visual acuity continues to remain 0.2 with no recurrence of CMO (Figure 2).

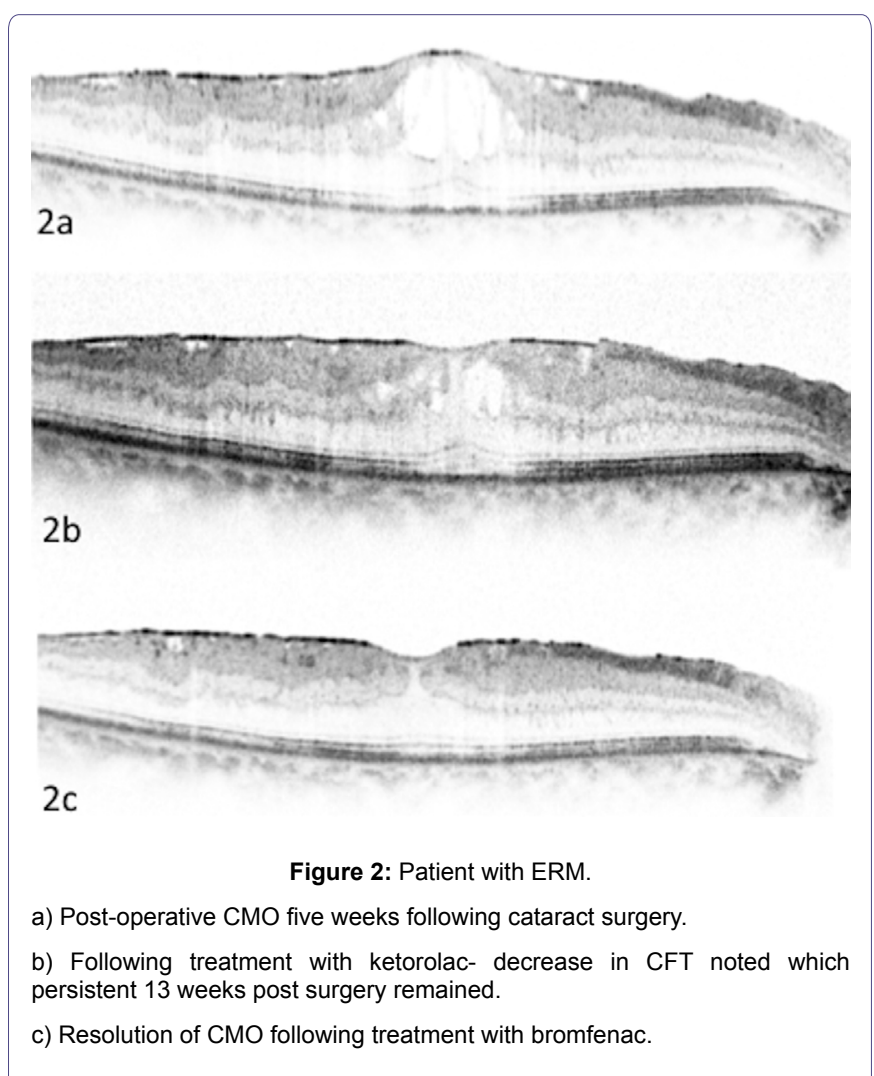

Case 5: A 59-year-old male developed recurrent CMO following cataract surgery. He gave previous history of having undergone retinal detachment repair in the same eye. On three occasions, CMO had been successfully treated with ketorolac eye drops but continued to recur after a six to eight week period. At the fourth recurrence, it was decided to switch from ketorolac to bromfenac twice a day. Four weeks later, CMO showed signs of resolution and his visual acuity improved. At five months follow up, there has been no recurrence of $\mathrm{CMO}$ and visual acuity continues to remain stable.

Case 6: A 62-year-old male underwent routine cataract surgery in his better eye (the other eye was densely amblyopic) and developed CMO six weeks later. His visual acuity dropped from LogMAR 0.0 to 0.5 (CFT-391 $\mu \mathrm{m})$. He was started on ketorolac eye drops along with topical steroids and six weeks later, his vision improved to 0.3. OCT showed a slight reduction in CMO. As the patient was extremely anxious, this being his only seeing eye, it was decided to substitute ketorolac with bromfenac drops and four weeks later, his vision improved to 0.0 with complete resolution of CMO (CFT-198 $\mu \mathrm{m})$. At four months follow up, no recurrence of CMO has been noted.

\section{Discussion}

Clinically significant CMO is defined as Snellen visual acuity of 20/40 (LogMAR 0.4) or worse. CMO is the most common cause of 
poor visual outcome following cataract surgery and the incidence is highest four to six weeks after surgery [3].

Although the exact pathogenesis of post-operative CMO remains unclear, it is postulated that surgical trauma to the intraocular tissues causes rupture of the blood aqueous barrier stimulating release of arachidonic acid thereby activating Cyclooxygenase (COX) enzymes resulting in the production of prostaglandins. Of the COX enzymes, COX-1 and 2 are known to be the main isoforms $[4,5]$.

Complicated cataract surgery, diabetes, previous CMO, previous retinal vein occlusion and epiretinal membrane are risk factors in the development of CMO, but there is insufficient evidence to warrant the use of prophylactic NSAIDs in these patients [6]. In our case series, two patients had epiretinal membrane diagnosed prior to undergoing cataract surgery and one patient underwent complicated phacoemulsification with anterior vitrectomy, placing them at a higher risk of developing CMO.

There is sufficient evidence showing the efficacy of ketorolac for the management of post-operative CMO and it is a widely used agent [7]. Bromfenac is a relatively new topical NSAID and experimental studies have proven bromfenac to have a greater penetration to the posterior segment than other ocular preparations [8]. Its other advantage is twice daily dosing, improving patient adherence and compliance [9]. Ketorolac on the other hand, has the advantage of being cost effective compared to bromfenac.

To date, there is little evidence that more expensive agents such as bromfenac have superiority compared with ketorolac for the management of acute $\mathrm{CMO}$ and as a result, ketorolac continues to remain the most widely used first line medical therapy for the management of post-operative acute CMO [2,6,7].

The mechanism of action of ketorolac and bromfenac is different in terms of their ability to inhibit COX-1 and COX-2 enzymes respectively. Ketorolac has a more potent COX-1 inhibitory activity where as Bromfenac and Nepafenac inhibit COX-2 selectively [9]. Predominantly higher levels COX-2 have been found in the retina during an increase in the inflammatory process and we postulate that this may be one of the reasons where switched therapy might be effective in resistant cases of CMO. Further randomized controlled trials are required to support this theory.

In our series of six cases, post-operative CMO was initially treated with ketorolac in combination with topical steroids. Four of these cases (cases 1-4) failed to show any improvement with ketorolac but showed a significant response to bromfenac. In case 5, the response to ketorolac was unsustained but after instating bromfenac, a sustained and complete resolution of CMO was noted. In case 6 , an incomplete response to ketorolac was seen after six weeks, but with bromfenac, the patient reported a rapid response. In our case series, there were no adverse events recorded in any of these patients.

Qi-Wei Wang et al., [10] evaluated the safety and efficacy of topical bromfenac following cataract surgery and compared inflammatory reactions and retinal foveal thickness with fluoromethalone or topical dexamethasone. Their results showed a significant anti-inflammatory response to bromfenac and they recommended using the drug instead of topical steroids following cataract surgery. Other studies have shown that bromfenac is a favourable drug and a valuable addition to the NSAID armamentarium for the management of CMO [11-13].
Bromfenac is a favourable treatment option in patients with $\mathrm{CMO}$ as a second line drug. We are in agreement with a review article by Shelsta et al., [6] where the authors recommend the use of a more expensive topical NSAID if no improvement in CMO is seen with a less costly NSAID such as ketorolac after four to six weeks. To our knowledge, this is the first case-series to demonstrate efficacy of switched NSAID therapy (from ketorolac to bromfenac) for the management of post-operative CMO. We recommend substituting ketorolac with another NSAID with a different mechanism of action prior to considering any invasive intraocular procedures.

\section{Conclusion}

Our case series has shown that switching from ketorolac to bromfenac with a different mechanism of action can be effective in resolving persistent post-operative $\mathrm{CMO}$ and may prevent the need for invasive treatment.

\section{References}

1. Heier JS, Topping TM, Baumann W, Dirks MS, Chern S (2000) Ketorolac versus prednisolone versus combination therapy in the treatment of acute pseudophakic cystoid macular edema. Ophthalmology 107: 2034-2038.

2. Levin DS, Lim Jl (2002) Update on pseudophakic cystoid macular edema treatment options. Ophthalmol Clin North Am 15: 467-472.

3. Agostini HT, Hansen LL, Feltgen N (2007) [Treatment of pseudophakic cystoid macular edema]. Ophthalmologe 104: 425-426, 428-430.

4. Lobo C (2012) Pathogenesis of Pseudophakic Cystoid Macular Oedema. European Ophthalmic Review 6: 178-184.

5. Kida T, Kozai S, Takahashi H, Isaka M, Tokushige H, et al. (2014) Pharmacokinetics and efficacy of topically applied nonsteroidal anti-inflammatory drugs in retinochoroidal tissues in rabbits. PLoS One 9: 96481.

6. Shelsta HN, Jampol LM (2011) Pharmacologic therapy of pseudophakic cystoid macular edema: 2010 update. Retina 31: 4-12.

7. Flach AJ, Dolan BJ, Irvine AR (1987) Effectiveness of ketorolac tromethamine $0.5 \%$ ophthalmic solution for chronic aphakic and pseudophakic cystoid macular edema. Am J Ophthalmol 103: 479-486.

8. Waterbury LD, Silliman D, Jolas T (2006) Comparison of cyclooxygenase inhibitory activity and ocular anti-inflammatory effects of ketorolac tromethamine and bromfenac sodium. Curr Med Res Opin 22: 1133-1140.

9. Jones J, Francis P (2009) Ophthalmic utility of topical bromfenac, a twice-daily nonsteroidal anti-inflammatory agent. Expert Opin Pharmacother 10: 23792385.

10. Wang QW, Yao K, Xu W, Chen PQ, Shentu XC, et al. (2013) Bromfenac sodium $0.1 \%$, fluorometholone $0.1 \%$ and dexamethasone $0.1 \%$ for control of ocular inflammation and prevention of cystoid macular edema after phacoemulsification. Ophthalmologica 229: 187-194.

11. Rajpal RK, Ross B, Rajpal SD, Hoang K (2014) Bromfenac ophthalmic solution for the treatment of postoperative ocular pain and inflammation: safety, efficacy, and patient adherence. Patient Preference and Adherence 8: 925931.

12. Donnenfeld ED, Holland EJ, Stewart RH, Gow JA, Grillone LR, et al. (2007) Bromfenac ophthalmic solution $0.09 \%$ (Xibrom) for postoperative ocular pain and inflammation. Ophthalmology 114: 1653-1662.

13. Cho H, Wolf KJ, Wolf EJ (2009) Management of ocular inflammation and pain following cataract surgery: focus on bromfenac ophthalmic solution. Clin Ophthalmol 3: 199-210. 